\title{
Correction to: The Quest for the Ultimate Anisotropic Banach Space
}

\author{
Viviane Baladi ${ }^{1}$
}

Published online: 16 February 2018

(C) Springer Science+Business Media, LLC, part of Springer Nature 2018

\section{Correction to: J Stat Phys (2017) 166:525-557 https://doi.org/10.1007/s10955-016-1663-0}

We provide corrections and complements to [1, §4]: Formulas (29) and, especially, (31) must be amended, as explained in Sects. 3 and 4 below. An argument to replace the $C^{r}$ norm of the weight by a supremum was missing at the end of the proof of Theorem 4.1 (see Sect. 2 below). Sections 1, 5, and 6 below give minor clarifications. None of the main statements are changed, except that:

- In Lemma 4.2 and Theorem 4.1, the condition $-(r-1)<s<-t<0$ must be replaced by $t-(r-1)<s<-t<0$. (See Sect. 4 below.)

- The bound (25) on the essential spectral radius in Theorem 4.1 is only proved up to an (arbitrarily small) $\epsilon>0$ and for a "zoomed" version of the space $\mathcal{U}_{1}^{t, s}(R)$ for some large $R(\epsilon)$. (See (1) in Sect. 2 below.)

\section{Details for the Leafwise Young Inequality (38)}

To prove (38) on p. 542, notice that, for $\ell \geq 1$ and $x \in \mathbb{R}^{d_{s}}$, Fubini implies

$$
\begin{aligned}
& \left(\psi_{\ell}^{\left(d_{s}\right)}\right)^{O p}\left[(\varphi * \hat{\psi}) \circ \pi_{\Gamma}^{-1}\right](x) \\
& \quad=\left[\left(\psi_{\ell}^{\left(d_{s}\right)}\right)^{O p} \int \hat{\psi}(z) \varphi\left(\pi_{\Gamma}^{-1}(\cdot)-z\right) \mathrm{d} z\right](x)
\end{aligned}
$$

I thank M. Jézéquel, who found several unclear points in [1] and helped to clarify them.

The original article can be found online at https://doi.org/10.1007/s10955-016-1663-0.

Viviane Baladi

viviane.baladi@imj-prg.fr

1 Institut de Mathématiques de Jussieu (IMJ-PRG), Sorbonne Université, CNRS, 4, Place Jussieu, 75005 Paris, France 


$$
\begin{aligned}
& =\left[\left(\psi_{\ell}^{\left(d_{s}\right)}\right)^{O p} \int \hat{\psi}(z) \varphi\left(\pi_{\Gamma-z}^{-1}(\cdot)\right) \mathrm{d} z\right](x) \\
& =\int_{\mathbb{R}^{n}} \hat{\psi}(z)\left(\psi_{\ell}^{\left(d_{s}\right)}\right)^{O p}\left[\varphi \circ \pi_{\Gamma-z}^{-1}\right](x) \mathrm{d} z .
\end{aligned}
$$

Since $\left\|\int_{\mathbb{R}^{n}} \hat{\psi}(z) \Phi_{z}(\cdot) \mathrm{d} z\right\|_{L_{p}\left(\mathbb{R}^{d_{s}}\right)} \leq\|\hat{\psi}\|_{L_{1}\left(\mathbb{R}^{d}\right)} \sup _{z}\left\|\Phi_{z}(\cdot)\right\|_{L_{p}\left(\mathbb{R}^{d_{s}}\right)}$ by the Minkowski integral inequality, we find,

$$
\left\|\left(\psi_{\ell}^{\left(d_{s}\right)}\right)^{O p}\left[(\varphi * \hat{\psi}) \circ \pi_{\Gamma}^{-1}\right]\right\|_{L_{p}\left(\mathbb{R}^{d_{s}}\right)} \leqslant\|\hat{\psi}\|_{L_{1}\left(\mathbb{R}^{d}\right)} \sup _{\tilde{\Gamma} \in \mathcal{F}}\left\|\left(\psi_{\ell}^{\left(d_{s}\right)}\right)^{O p}\left[\varphi \circ \pi_{\tilde{\Gamma}}^{-1}\right]\right\|_{L_{p}\left(\mathbb{R}^{d_{s}}\right)} .
$$

\section{Proof of Theorem 4.1}

The bound (43) is correct as stated, but to prove Theorem 4.1 we would need it for weights $\tilde{f}_{m}(x)=\prod_{j=0}^{m-1}\left(\theta_{\vec{\omega}}^{(m)} g\left(T^{-j}(x)\right)\right.$, where $\sum_{\vec{\omega}} \theta_{\vec{\omega}}^{(m)} \equiv 1$ is a smooth partition of unity adapted to $T^{m}$ (independently of $\Gamma$ ), taking into account the fact that minimal sub-covers are needed for thermodynamic estimates. The bound (43) does not seem to always hold for such $\tilde{f}_{m}$. Hence, Theorem 4.1 is only proved if we add (arbitrarily small) $\epsilon>0$ to the expression (25), and if we replace $\mathcal{U}_{1}^{t, s}$ by its $R$-zoomed version $\mathcal{U}_{1}^{t, s}(R)($ as in [3, §2.3]), for $R$ depending on $\epsilon$ :

We recall the construction in [3, §2.3]. Our charts are $\kappa_{\omega}: U_{\omega} \rightarrow V_{\omega} \subset M$ with $U_{\omega} \in$ $\mathbb{R}^{d}$ and $\omega \in \Omega$. Fix a $C^{\infty}$ function $\alpha: \mathbb{R}^{d} \rightarrow[0,1]$ with $\alpha(z)=0$ if $|z| \geq d$, and $\sum_{m \in \mathbb{Z}^{d}} \alpha(z-m)=1$. For $R \geq 1$ and

$$
(\omega, m) \in \mathcal{Z}(R):=\left\{(\omega, m) \mid \omega \in \Omega, m \in\left(R \cdot U_{\omega}\right) \cap \mathbb{Z}^{d}\right\},
$$

define $\hat{\alpha}_{\omega, m}^{R}: M \rightarrow[0,1]$ by $\hat{\alpha}_{\omega, m}^{R}(x)=0$ if $x \notin V_{\omega}$, and

$$
\hat{\alpha}_{\omega, m}^{R}(x)=\alpha\left[R \cdot\left(\kappa_{\omega}^{-1}(x)\right)-m\right], \quad \forall x \in V_{\omega} .
$$

This gives a partition of unity in the sense that $\sum_{m \in\left(R \cdot U_{\omega}\right) \cap \mathbb{Z}^{d}} \hat{\alpha}_{\omega, m}^{R}(x)=1$ for all $x \in V_{\omega}$. (The intersection multiplicity of this partition of unity is bounded, uniformly in $R$.) Finally, fixing $s, t \in \mathbb{R}$ and $R \geq 1$, we write $\kappa_{\omega}^{R}(z)=\kappa_{\omega}(z / R)$, and we set for $\varphi \in L^{\infty}(M),{ }^{1}$

$$
\|\varphi\|_{\mathcal{U}_{1}^{t, s}(R)}=\sum_{(\omega, m) \in \mathcal{Z}(R)}\left\|\left(\hat{\alpha}_{\omega, m}^{R} \cdot \varphi\right) \circ \kappa_{\omega}^{R}\right\|_{\mathcal{U}_{1}^{t, s}} \leq \infty
$$

The space $\mathcal{U}_{p}^{t, s}(R)$ is the closure of $L^{\infty}(M)$ for the norm $\|\varphi\|_{\mathcal{U}_{p}^{t, s}(R)}$.

Since $\left\|\tilde{f}_{m}^{R}\right\|_{C^{r}} \leq 2 \sup \left|\tilde{f}_{m}^{R}\right|$, for $\tilde{f}_{m}(z)=\tilde{f}_{m}(z / R)$, if $R \geq 1$ is large enough (depending on $m$ ), we may then prove Theorem 4.1 up to adding $\epsilon>0$ to (25) and replacing $\mathcal{U}_{1}^{t, s}$ by $\mathcal{U}_{1}^{t, s}(R)$ for suitable $R(\epsilon)$.

Finally, the last sentence in the proof of Theorem 4.1 on p. 543 must be shortened to: "We just mention here that, in the present case, the "fragmentation lemma" (used to expand along a partition of unity) is just the triangle inequality, while the "reconstitution lemma" (used to regroup the terms from a partition of unity) is the trivial inequality $\sum\left|a_{k} e_{k}\right| \leq$ $\left(\sum\left|a_{k}\right|\right)$ sup $\left|e_{k}\right|$. ." Footnote 18 on the same page must be suppressed.

$\overline{1}$ Note that for fixed $R$, the sum in (1) involves a uniformly bounded number of terms. 


\section{Details for (40) in Sublemma 4.4 and Correcting (29)}

We explain how to bound $\|f(\varphi \circ F)\|_{p, \Gamma}^{s}$, by duality, giving the proof of (40) on p. 543: Since $B_{p, \infty}^{s}$ is the dual of $b_{p^{\prime}, 1}^{|s|}$ (with $1 / p^{\prime}=1-1 / p$ ), setting $F_{\Gamma}=\pi_{F(\Gamma)} \circ F \circ \pi_{\Gamma}^{-1}$, it suffices to estimate $\left\|\left((f h) \circ F_{\Gamma}^{-1}\right)\left|\operatorname{det}\left(D F_{\Gamma}\right)^{-1}\right|\right\|_{B_{p^{\prime}, 1}^{|s|}\left(\mathbb{R}^{d_{s}}\right)}$ for $C^{\infty}$ functions $h$. First note that $\left\|\left((f h) \circ F_{\Gamma}^{-1}\right)\left|\operatorname{det}\left(D F_{\Gamma}\right)^{-1}\right|\right\|_{L_{p^{\prime}}\left(\mathbb{R}^{d_{s}}\right)} \leq \sup \frac{|f|}{\left|\operatorname{det} D F_{\Gamma}\right|^{1 / p}}$. The $B_{p^{\prime}, 1}^{|s|}\left(\mathbb{R}^{d_{s}}\right)$ norm of $v$ is equivalent to $^{2}$

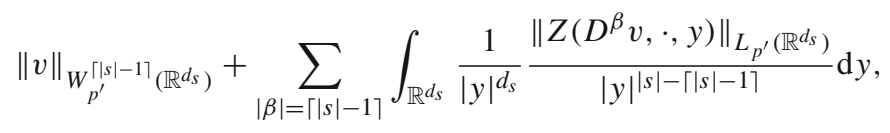

where $\|v\|_{W_{p^{\prime}}^{k}}=\sum_{0 \leq|\beta| \leq k}\left\|D^{\beta} v\right\|_{L_{p^{\prime}}}$ and $Z(w, x, y)=w(x+y)+w(x-y)-2 w(x)$. Thus, since inf $\left|D F_{\Gamma}\right| \geq\|F\|_{-} \geq 1$, and using the "Zygmund derivation" in ${ }^{3}[4, \S 2]$

$$
\begin{aligned}
Z(f h, x, y)= & f(x) Z(h, x, y)+h(x) Z(f, x, y) \\
& +\Delta_{+}(f, x, y) \Delta_{+}(h, x, y)+\Delta_{-}(f, x, y) \Delta_{-}(h, x, y),
\end{aligned}
$$

where $\Delta_{+}(v, x, y)=(v(x+y)-v(x))$ and $\Delta_{-}(v, x, y)=(v(x)-v(x-y))$, and recalling that for any noninteger $\sigma>0$ [6, Props 2.1.2, 2.2.1]

$$
\|v\|_{\mathcal{W}_{p^{\prime}, p^{\prime}}^{\sigma}} \leq C\left(p^{\prime}, \sigma\right)\|v\|_{B_{p^{\prime}, p^{\prime}}^{\sigma}} \leq C^{2}\left(p^{\prime},|s|\right)\|v\|_{B_{p^{\prime}, 1}^{\sigma}},
$$

(with $\mathcal{W}_{p^{\prime}, p^{\prime}}^{\sigma}$ the Slobodeckij norm), we find for any $\epsilon>0$ constants $C(\mathcal{F})$ and $C(\mathcal{F}, \epsilon)$ so that

$$
\begin{aligned}
& \left\|\left((f h) \circ F_{\Gamma}^{-1}\right)\left|\operatorname{det}\left(D F_{\Gamma}\right)^{-1}\right|\right\|_{B_{p^{\prime}, 1}^{|s|}\left(\mathbb{R}^{d_{s}}\right)} \\
& \leq C(\mathcal{F}) \sum_{j=0}^{\lceil|s|-1\rceil} \sum_{\ell=0}^{j} \frac{1}{\|F\|_{-}^{\ell}} \frac{1}{\left|\operatorname{det} D F_{\Gamma}\right|^{1 / p}}\|h\|_{W_{p^{\prime}}^{\ell}} \\
& \times \sum_{i=0}^{j-\ell}\left\|f \circ F_{\Gamma}^{-1}\right\|_{C^{i}}\left\|\left|\operatorname{det}\left(D F_{\Gamma}^{-1}\right)\right|\right\|_{C^{j-\ell-i}}\left\|D F_{\Gamma}^{-1}\right\|_{C^{j-\ell-i}} \\
& +C(\mathcal{F}) \sum_{\ell=1}^{\lceil|s|-1\rceil} \frac{1}{\|F\|_{-}^{\ell}} \frac{1}{\left|\operatorname{det} D F_{\Gamma}\right|^{1 / p}}\|h\|_{B_{p^{\prime}, 1}^{\ell}} \\
& \times \sum_{i=0}^{\lceil|s|-1\rceil-\ell}\left\|f \circ F_{\Gamma}^{-1}\right\|_{C^{i}}\left\|\left|\operatorname{det}\left(D F_{\Gamma}^{-1}\right)\right|\right\|_{C\lceil|s|-1\rceil-\ell-i}\left\|D F_{\Gamma}^{-1}\right\|_{C\lceil|s|-1\rceil-\ell-i} \\
& +C(\mathcal{F}, \epsilon) \frac{\left\|D F_{\Gamma}^{-1}\right\|_{C^{\epsilon}}}{\|F\|_{-}^{|s|-2 \epsilon}} \frac{\left\|\left|\operatorname{det}\left(D F_{\Gamma}^{-1}\right)\right|\right\|_{C^{\epsilon}}}{\left|\operatorname{det} D F_{\Gamma}\right|^{1 / p}}\|h\|_{B_{p^{\prime}, 1}^{|s|-\epsilon}}\left\|f \circ F_{\Gamma}^{-1}\right\|_{C^{\epsilon}} \\
& +C(\mathcal{F}, \epsilon) \frac{1}{\|F\|_{-}^{|s|}} \frac{\sup |f|}{\left|\operatorname{det} D F_{\Gamma}\right|^{1 / p}}\|h\|_{B_{p^{\prime}, 1}^{|s|}} .
\end{aligned}
$$

\footnotetext{
${ }^{2}$ See e.g. [6, §2.1], with $\lceil x\rceil$ the smallest integer which is $\geq x$.

${ }^{3}$ See also [4, (2.6)-(2.8)], writing $\left|f_{i}^{\prime}\right|_{\delta}=\left|f_{i}^{\prime}\right|\left|f_{i}^{\prime}\right|_{\delta} /\left|f_{i}^{\prime}\right|$ in [4, (2.5)], and noting that $\left|\left(F_{\Gamma}^{-1}\right)^{\prime}\right|_{\delta}=$ $\left|F_{\Gamma}^{\prime}\right| \delta /\left|F_{\Gamma}^{\prime}\right|^{2}$ so that $\left|\left(F_{\Gamma}^{-1}\right)^{\prime}\right|_{\delta} /\left|\left(F_{\Gamma}^{-1}\right)^{\prime}\right|=\left|F_{\Gamma}^{\prime}\right| \delta /\left|F_{\Gamma}^{\prime}\right|$. 


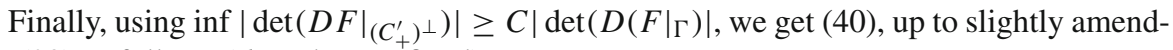
ing (29) as follows ( $d_{s}$ and $p$ are fixed):

$$
C(F, \Gamma, s)=C^{\prime}(\mathcal{F})|s|\left\|D\left(\left.F\right|_{\Gamma}\right)^{-1}\right\|_{C^{r-1}}\left\|\left|\operatorname{det}\left(D F_{\Gamma}^{-1}\right)\right|\right\|_{C^{r-1}} .
$$

Similarly, in (42) one should replace $\sup _{\Gamma}\left\|f \circ F^{-1}\right\|_{C^{r-1}(F(\Gamma))}$ by

$$
\sup _{\Gamma}\left\|f \circ F^{-1}\right\|_{C^{r-1}(F(\Gamma))}\left\|\left|\operatorname{det}\left(D\left(F_{\left(C_{+}^{\prime}\right)^{\perp}}\right)^{-1}\right)\right|\right\|_{C^{r-1}(F(\Gamma))} .
$$

\section{Bounding $\left\|H_{n, \sigma}^{\ell, \tau}(v)\right\|_{p, \Gamma}^{s}($ Proof of Lemma 4.2): Correcting (31)}

Since $\|\cdot\|_{p, \Gamma}^{s}$ is not an $L_{p}$ norm, (38) does not suffice to deduce from (54) a bound on $\left\|H_{n, \sigma}^{\ell, \tau}(v)\right\|_{p, \Gamma}^{s}$. For any compact $K \subset \mathbb{R}^{d}$ and any $\delta>0$, there exists $C_{0} \geq 2$ so that for all $C_{0}^{\prime} \geq C_{0}$ there exists $\widetilde{C}_{0}$ so that for all $v$ supported in $K$,

$$
\begin{aligned}
& \left\|\left(\psi_{\ell}^{O p} v\right) \circ \pi_{\widetilde{\Gamma}}^{-1}\right\|_{L_{p}\left(\mathbb{R}^{d_{s}}\right)} \\
& \leq \widetilde{C}_{0} 2^{\ell(-s+\delta)} \sum_{j=0}^{\ell+\left[C_{0}^{\prime}\right]} 2^{j(s-\delta)} \|\left(\psi_{j}^{d_{s}}\right)^{O p}\left(\left(\psi_{\ell}^{O p} v\right) \circ \pi_{\widetilde{\Gamma}^{-1}}^{O p} \|_{L_{p}\left(\mathbb{R}^{d_{s}}\right)}\right. \\
& \quad+C_{0} \sum_{j=\ell+\left[C_{0}^{\prime}\right]+1}^{\infty} 2^{-j r} \sum_{m=\ell-2}^{\ell+2} \sup _{\widehat{\Gamma}}\left\|\left(\psi_{m}^{O p} v\right) \circ \pi_{\widehat{\Gamma}}^{-1}\right\|_{L_{p}\left(\mathbb{R}^{d_{s}}\right)}, \forall \ell, \quad \forall \widetilde{\Gamma} .
\end{aligned}
$$

(This is clear if $\widetilde{\Gamma}$ is ${ }^{4}$ affine, otherwise, proceed as in [2, Lemma 3.5], using the $L_{p}$ version of the leafwise Young inequality [5, Lemma 4.2], to obtain the above estimate in the sum over $j>\ell+C_{0}^{\prime}$, after decomposing $v=\sum_{m} \psi_{m}^{O p} v$ and using almost orthogonality.) Therefore, since $|s|<r$ and $a \leq b+\epsilon a$ implies $a \leq(1-\epsilon)^{-1} b$ if $a>0, b>0$, and $\epsilon<1$, for each $\delta>0$ there is $C$ so that

$$
2^{\ell t}\left\|\left(\psi_{\ell}^{O p} v\right) \circ \pi \widetilde{\Gamma}^{-1}\right\|_{L_{p}\left(\mathbb{R}^{d_{s}}\right)} \leq C 2^{\ell(-s+\delta)}\|v\|_{\mathcal{U}_{p}^{t, s}} \quad \forall \widetilde{\Gamma}, \quad \forall \ell .
$$

Then, applying $\|\phi\|_{B_{p, \infty}^{s}} \leq C\|\phi\|_{L_{p}}$ to $\phi=\left(H_{n, \sigma}^{\ell, \tau}\left(\sum_{i=-2}^{2} \psi_{\ell+i}^{O p} v\right)\right) \circ \pi_{\Gamma}^{-1}$, and using (54) and the $L_{p}$ version of [5, Lemma 4.2], one obtains

$2^{\ell t}\left\|H_{n, \sigma}^{\ell, \tau}(v)\right\|_{p, \Gamma}^{s}=2^{\ell t}\left\|H_{n, \sigma}^{\ell, \tau}\left(\sum_{i=-2}^{2} \psi_{\ell+i}^{O p} v\right)\right\|_{p, \Gamma}^{s} \leq C_{F, f} 2^{-(r-1) \max \{n, \ell\}} 2^{(-s+\delta) \ell}\|v\|_{\mathcal{U}_{p}^{t, s}}$.

This replaces the stronger bound stated two lines above (52) on p. 546 and gives the following weakening of (31):

$$
\left\|\left(\phi-\mathcal{R}_{n_{0}}\right) \mathcal{M}_{c} \varphi\right\|_{\mathcal{U}_{p}^{\mathbf{C}_{ \pm}^{\prime}, t, s}} \leq C_{F, f, \delta} 2^{-(r-1-2 \delta-(t-s)) n_{0}} .
$$

Therefore, one must replace $-(r-1)<s<-t<0$ by $t-(r-1)<s<-t<0$ in Lemma 4.2, and thus in ${ }^{5}$ Theorem 4.1.

\footnotetext{
4 The second line is then not needed.

5 The condition on $s$ and $t$ was not explicited in Theorem 4.1.
} 


\section{Fixing the End of the Proof of Sublemma 4.4}

The formulas for some kernels in the proof of Sublemma 4.4 (p. 548) are garbled. The corrections are detailed below. The statement of the sublemma is unchanged.

Lines 9-14 and the footnote of p. 548 must be replaced by "Recalling the functions $b_{m}$ from (53), we claim that there exists a constant $C_{0}>1$ depending only on $C_{\mathcal{F}}$ and $\mathbf{C}_{ \pm}$so that, for any $\Gamma \in \mathcal{F}\left(\mathbf{C}_{+}\right)$and all $n, n_{s}$, the kernels $V_{n_{s}, \Gamma}^{n,-}(w, y)$ defined for $w \in \Gamma$ and $y \in \mathbb{R}^{d}$ by

$$
\int_{\mathbb{R}^{d}} \mathbb{F}^{-1}\left(\psi_{\Theta^{\prime}, n,-}\right)(-x)\left(\phi \cdot \psi_{n_{s}}^{O p(\Gamma+x)} \tilde{\varphi}\right)(w+x) \mathrm{d} x=\frac{1}{(2 \pi)^{d+d_{s}}} \int_{\mathbb{R}^{d}} V_{n_{s}, \Gamma}^{n,-}(w, y) \tilde{\varphi}(y) \mathrm{d} y
$$

satisfy, ${ }^{21}$

$$
\left|V_{n_{s}, \Gamma}^{n,-}(w, y)\right| \leq C_{0} 2^{-(r-1) n} b_{n_{s}}(w-y) \quad \text { if } C_{0} 2^{n_{s}} \leq 2^{n} \text { or } 2^{n_{s}} \geq C_{0} 2^{n} . "
$$

Replace lines $15-19$ of p. 548 by: "To prove (60), recall (16) and note that

$$
\begin{aligned}
V_{n_{s}, \Gamma}^{n,-}(w, y)= & \int_{\eta \in \mathbb{R}^{d}, x_{-}, \eta_{s} \in \mathbb{R}^{d_{s}}} e^{-i x\left(y, x_{-}\right) \eta} e^{i\left(\pi_{\Gamma+x\left(y, x_{-}\right)}\left(w+x\left(y, x_{-}\right)\right)-z\left(y, x_{-}\right)\right) \eta_{s}} \\
& \times \frac{\phi\left(w+x\left(y, x_{-}\right)\right)}{\left|\operatorname{det} D \mathcal{Y}_{\Gamma, x_{-}}\left(\mathcal{Y}_{\Gamma, x_{-}}^{-1}(y)\right)\right|} \psi_{n_{s}}^{\left(d_{s}\right)}\left(\eta_{s}\right) \psi_{\Theta^{\prime}, n,-}(\eta) \mathrm{d} \eta \mathrm{d} \eta_{s} \mathrm{~d} x_{-},
\end{aligned}
$$

using for each $x_{-} \in \mathbb{R}^{d_{s}}$ the $C^{r}$ change of variable $y=\mathcal{Y}_{\Gamma, x_{-}}\left(z, x_{+}\right):=\pi_{\Gamma+\left(x_{-}, x_{+}\right)}^{-1}(z)$ in $\mathbb{R}^{d}$, with $z \in \mathbb{R}^{d_{s}}$ and $x_{+} \in \mathbb{R}^{d_{u}}$, setting also

$$
x\left(y, x_{-}\right)=\left(x_{-}, \Pi_{+}\left(\mathcal{Y}_{\Gamma, x_{-}}^{-1}(y)\right)\right), \quad z\left(y, x_{-}\right)=\pi_{\Gamma+x\left(y, x_{-}\right)}(y),
$$

where $\Pi_{+}: \mathbb{R}^{d_{s}+d_{u}} \rightarrow \mathbb{R}^{d_{u}}$ is defined by $\Pi_{+}\left(x_{-}, x_{+}\right)=x_{+}$. Next just like in [10,11] (see also [Lemma 2.34, 2]), using that $\pi_{\Gamma+x}(w+x)=\pi_{\Gamma}(w)+x_{-}$if $x=\left(x_{-}, x_{+}\right) \in \mathbb{R}^{d_{s}} \times \mathbb{R}^{d_{u}}$ and that $\Gamma \in \mathcal{F}$, first integrate by parts (see Appendix 3) $(r-1)$ times with respect to $x_{-} \in \mathbb{R}^{d_{s}}$ in the formula for $V_{n_{s}, \Gamma}^{n,-}(w, y)$, and second, noticing that $\|y-w\|>\epsilon$ implies that either $\left\|\pi_{\Gamma}(w)-\pi_{\Gamma+x\left(y, x_{-}\right)}(y)\right\|>\epsilon /\left(2 C_{0}\right)$ or $\left\|\Pi_{+}\left(\mathcal{Y}_{\Gamma, x_{-}}^{-1}(y)\right)\right\|>\epsilon /\left(2 C_{0}\right)$, integrate by parts with respect the other variables as many times as necessary. It is an enlightening exercise to prove (60) for affine $\Gamma$."

There is a minor typo in the left-hand side of (64) on p. 549, which should read:

$$
\left\|\int \mathbb{F}^{-1}\left(\psi_{\Theta^{\prime}, n,-}\right)(-x) \cdot\left(\mathcal{R}_{\tilde{n}_{s}, \Gamma+x}\right)(\tilde{\varphi})(\cdot+x) \mathrm{d} x\right\|_{p, \Gamma}^{s} \leq \sup _{x} C_{1} 2^{-(r-1) m_{0}}\|\tilde{\varphi}\|_{p, \Gamma+x}^{s} .
$$

Finally, (64) follows from (60) and the leafwise Young inequality (38).

\section{Typos}

On p. 537, the condition $\mathbb{R}^{d_{s}} \times\{0\} \subset \mathbf{C}_{-}$must be replaced by " $\mathbb{R}^{d_{s}} \times\{0\}$ is included in $\left(\mathbb{R}^{d} \backslash \mathbf{C}_{+}\right) \cup\{0\}$ " (thrice, including Defs 3.2-3.3). Also, the assumptions ensure that $\Pi_{\Gamma}$

${ }^{21}$ For the kernels $V_{n_{s}, \Gamma+x}^{n,+}(w, y)$ defined by replacing $\psi_{\Theta^{\prime}, n,-}$ with $\psi_{\Theta, n,+}$, we only get $C_{0}>1$ so that $\left|V_{n_{s}, \Gamma}^{n,+}(w, y)\right| \leq C_{0} 2^{-(r-1) n} b_{n_{s}}(w-y)$ if $C_{0} 2^{n_{s}} \geq 2^{n}$. In particular, $V_{n_{s}, \Gamma}^{n,+}$ need not be small if $n$ is big and $n_{s}$ small. 
is surjective. Same page, 6 lines after (17), the norms are equivalent uniformly in $\Gamma$, not equal, due to the Jacobian. In Lemma 4.2, one must assume that $F$ can be extended by a bilipschitz regular cone hyperbolic diffeomorphism $\tilde{F}$ of $\mathbb{R}^{d}$, with $\|\tilde{F}\|_{+}, 1 /\|\tilde{F}\|_{-}, 1 /\|\tilde{F}\|_{--}$

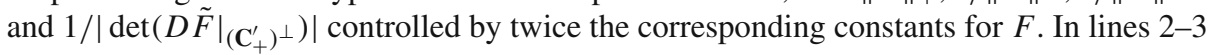
of p. 555, the sum is over all $\ell \geq 0$, and in line 2 , one of the $\left(1+\left\|x_{-}\right\|\right)^{Q_{1}}$ must be replaced by $\left(1+\left\|x_{+}\right\|\right)^{Q_{2}}$, while $\psi_{\ell}^{O p} v(x)$ should be replaced by $\left\|\psi_{\ell}^{O p} v\right\|_{L_{\infty}}$.

\section{References}

1. Baladi, V.: The quest for the ultimate anisotropic Banach space. J. Stat. Phys. 166, 525-557 (2017) (in honour of Ruelle and Sinai). https://doi.org/10.1007/s10955-016-1663-0

2. Baladi, V.: Characteristic functions as bounded multipliers on anisotropic spaces. Proc. Amer. Math. Soc. (to appear). arXiv:1704.00157

3. Baladi, V., Gouëzel, S.: Banach spaces for piecewise cone hyperbolic maps. J. Modern Dyn. 4, 91-135 (2010)

4. Baladi, V., Jiang, Y., Lanford, O.E.: Transfer operators acting on Zygmund functions. Trans. Am. Math. Soc. 348, 1599-1615 (1996)

5. Baladi, V., Tsujii, M.: Dynamical determinants and spectrum for hyperbolic diffeomorphisms. In: Probabilistic and Geometric Structures in Dynamics. Contemporary Mathematics, vol. 469. American Mathematical Society, Providence, pp. 29-68 (2008)

6. Runst, T., Sickel, W.: Sobolev Spaces of Fractional Order, Nemytskij Operators, and Nonlinear Partial Differential Equations. Walter de Gruyter \& Co., Berlin (1996) 